\title{
Adaptação de Interface de Objeto de Aprendizagem baseada nos Estilos de Aprendizagem: uma Avaliação da Qualidade Afetiva
}

\author{
Zenaide Carvalho da Silva ${ }^{1}$, Leandro R. Ferreira ${ }^{2}$, Andrey Ricardo Pimentel ${ }^{2}$ \\ ${ }^{1}$ Universidade Federal do Sul e Sudeste do Pará (UNIFESSPA) \\ Folha 17, Quadra 04, Lote Especial - 68505-080 - Marabá - PA - Brasil \\ ${ }^{2}$ Universidade Federal do Paraná (UFPR) \\ Caixa Postal 19.081 - 81.531-980 - Curitiba - PR - Brasil \\ zenaide.silva@unifesspa.edu.br, \{lrferreira, andrey\}@inf.ufpr.br
}

\begin{abstract}
The creation of new ways of presenting the contents of learning objects (LO) taking into account the learning style (LS) of the student can generate a greater motivation in the use of this type of educational resource. The most relevant characteristics of the $L S$ were mapped and associated, with the most appropriate ways to present the LO content, creating a presentation adaptation of the LO content considering the LS. An OA prototype with interface adapted from the created model was developed to evaluate the affective quality of the approaches used, through experiments with student. The results of the students' emotional responses to the adaptation are presented and discussed.
\end{abstract}

Resumo. A criação de novas formas de apresentação de conteúdos de objetos de aprendizagem (OA) levando em consideração o estilo de aprendizagem (EA) do aluno pode gerar uma motivação maior no uso desse tipo de recurso educacional. As características mais relevantes dos EA foram mapeadas e associadas, com as formas mais adequadas de apresentar o conteúdo do OA, criando uma adaptação de apresentação do conteúdo do OA considerando os EA. Um protótipo do OA com interface adaptada a partir do modelo criado foi desenvolvido para avaliação da qualidade afetiva das abordagens usadas, por meio de experimentos com alunos. Os resultados das respostas emocionais dos alunos em relação à adaptação criada são apresentados e discutidos.

\section{Introdução}

Os estilos de aprendizagem (EA) são as preferências dos alunos na forma de perceber, captar, organizar, processar e compreender a informação [Felder and Silverman 1988]. Cada estilo apresenta características específicas, as quais precisam ser identificadas e mapeadas para que seja possível, realizar a adaptação do material educacional às necessidades dos alunos. Nessa pesquisa o material educacional considerado foi o objeto de aprendizagem (OA) definido como qualquer entidade, digital ou não, que pode ser usada, reusada ou referenciada durante o aprendizado apoiado sobre a tecnologia [Wiley 2001].

$\mathrm{Na}$ realidade a maioria dos materiais educacionais disponíveis nos ambientes 
VII Congresso Brasileiro de Informática na Educação (CBIE 2018)

Anais do XXIX Simpósio Brasileiro de Informática na Educação (SBIE 2018)

virtuais de aprendizagem são definidos e criados, seguindo uma abordagem "one size fits all", ou seja, todos os alunos recebem o mesmo conteúdo e da mesma forma, portanto as suas preferências individuais de aprendizagem não são consideradas. Nesse caso se o processo de aprendizagem não for adequado ao estilo do aluno, provavelmente a sua motivação para utilizar material educacional pode diminuir e o seu desempenho como aluno poderá ser comprometido.

Nesse contexto esse trabalho apresenta uma avaliação da qualidade afetiva da adaptação de apresentação de conteúdo dos materiais educacionais, aqui definidos como $\mathrm{OA}$, utilizando as características e preferências dos EA como critério para adaptação de interface de OA, definido em [Silva et al. 2016]. Um protótipo de OA com a interface adaptada com base no EA, a partir do modelo de [Silva et al. 2016] foi criado para realizar avaliação da qualidade afetiva das abordagens usadas, por meio de experimentos com alunos. Os resultados obtidos das medidas subjetivas das respostas emocionais do aluno em relação a interface, demonstraram que a adaptação criada atingiu os resultados almejados, em relação a adequação da interface do OA, de acordo com os estilos do modelo de Felder-Silverman [Felder and Silverman 1988].

O texto está estruturado da seguinte forma. A seção 2 apresenta uma revisão sobre estilos de aprendizagem. A seção 3 apresenta a adaptação de interface de OAs considerando os EA definida em [Silva et al. 2016]. A seção 4 apresenta e discute os resultados da avaliação da qualidade afetiva da interface; e, por fim, na Seção 5, são feitas as considerações finais.

\section{Estilos de Aprendizagem (EA)}

A definição de EA mais comumente aceita é de [Keefe 1979], considera o EA uma composição de características cognitivas, afetivas e fatores fisiológicos que servem como indicadores relativamente estáveis de como um aluno percebe, interage e responde ao ambiente de aprendizagem.

Existem vários modelos de $\mathrm{EA}$, influenciados por diferentes teorias da psicologia da aprendizagem, vários desses modelos são discutidos no trabalho de [Coffield et al. 2004], e podem ser usados para representar os estilos dos alunos.

Esta pesquisa utilizou o modelo de Felder-Silverman, por ser considerado o mais indicado para ser utilizado em ambientes voltados para a educação, adequação melhor de suas escalas às características dos materiais de aprendizagem, além de ser mais utilizado no contexto nacional e internacional em pesquisas relativas à adaptação de materiais de aprendizagem, pois consegue prover um bom grau de adaptatividade aos perfis dos alunos [Aguiar et al. 2014, Dorça et al. 2013, Graf 2007, Valaski et al. 2011].

O modelo de Felder-Silverman foi desenvolvido pelo professor Richard M. Felder e pela psicóloga Linda K. Silverman, que classifica os alunos em número de escala de acordo com a forma com que cada aluno recebe e processa as informações. $\mathrm{O}$ modelo proposto classifica o aluno em quatro dimensões detalhadas em [Felder and Silverman 1988]: a) Percepção da Informação (Sensorial x Intuitivo); b) Retenção da informação (Visual x Verbal); c) Processamento da informação (Ativo x Reflexivo) e d) Organização da informação (Sequencial x Global).

Esse modelo utiliza como instrumento de mensuração o ILS (Index of Learning 
VII Congresso Brasileiro de Informática na Educação (CBIE 2018)

Anais do XXIX Simpósio Brasileiro de Informática na Educação (SBIE 2018)

Styles) para identificar os EA baseados em FSLSM (Felder and Silverman learning Style Model), que compreende quarenta e quatro perguntas, onze para cada uma das quatro dimensões anteriormente citadas, mais detalhes em [Felder and Soloman 2005].

\section{Adaptação de Interface do Objeto de Aprendizagem baseada no Estilo de Aprendizagem}

Foi realizado um levantamento das características dos oito estilos do modelo de FelderSilverman, para identificar aspectos importantes que poderiam ser utilizados na criação de uma associação dessas características com as formas mais adequadas de apresentar o conteúdo, para adaptar a interface de acordo com as preferências de cada estilo. Também foram investigados comportamentos/ações dos alunos quando os mesmos utilizavam um ambiente virtual de aprendizagem, os quais foram analisados para deduzir as características de apresentação para OA para os oito estilos do modelo de Felder-Silverman. Essa investigação baseou-se nos estudos de [Graf 2007, Sanders and Bergasa-Suso 2010, Hamada et al. 2011], entre outros autores.

As Características de Apresentação para OA foram definidas para compor uma associação com os elementos de interface para poder estabelecer uma apresentação mais planejada e expressada visual e funcionalmente do OA de acordo com cada estilo de aprendizagem. Considerando que o layout da interface é a arte de manipular a atenção do usuário a fim de transmitir um significado, sequência e pontos de interação, foram criados os seguintes elementos, baseados em [Tidwell 2010]:

- Elementos de controle: Comumente conhecidos como botões, menus, barras de navegação e ícones, são componentes básicos em qualquer interface gráfica de usuário. São eles que estabelecem o controle sobre o conteúdo e à navegação. No trabalho definimos a "área de controle de navegação" que estabelece os botões de navegação e a "árvore de conteúdos" que apresenta o índice de conteúdos em uma ordem previamente estabelecida, esses dois itens de controle servem para o aluno navegar nos conteúdos do OA.

- Elementos de composição: Organização dos componentes de forma a gerar sentido, ordem aos diferentes fatores visuais da interface, que em conjunto produz o efeito de harmonizar os componentes. No trabalho criamos a "área de conteúdo", o "índice de Conteúdos" e a "área de controle de navegação" onde fícam dispostos todos os componentes que constituem o OA.

- Elementos de conteúdo: Recursos formados por textos e imagens que são os itens que compõem o conteúdo do OA. São formados por vinte tipos de recursos que dependendo do estilo podem ser usados, como vídeo, figura, entre outros.

Com base nas características de apresentação para o OA em relação ao sequenciamento, à apresentação e à forma/formato de conteúdo e recursos, foi possível estabelecer os parâmetros e os atributos necessários para criar a adaptação de apresentação de OA com base nos EAs definidas em [Silva et al. 2016].

$\mathrm{Na}$ definição da estrutura de adaptação da apresentação do conteúdo do OA, consideramos que este é formado por elementos de composição de conteúdo de um OA, constituídos pelos seguintes itens: Resumo (Res), fornece uma visão geral e clara do conteúdo abordado; Introdução (Int), composta por um conteúdo breve para 
VII Congresso Brasileiro de Informática na Educação (CBIE 2018)

Anais do XXIX Simpósio Brasileiro de Informática na Educação (SBIE 2018)

apresentação do assunto a ser estudado de um domínio; Desenvolvimento (Des), composto por um conteúdo mais abrangente, que contempla o assunto de um domínio de forma mais completa; Atividade (Ati), formada por um conteúdo para fixação do assunto; e Avaliação (Ava), avaliação do conteúdo abordado de um domínio.

Os elementos de composição de conteúdo foram organizados em relação aos parâmetros e atributos definidos em [Silva et al. 2016], tendo como base o EA do aluno, e foram formalizados como:

a) forma de exploração (FE), define como o conteúdo pode ser explorado pelo aluno, podendo ser em rede (Red), que consiste em uma investigação mais aleatória, sem seguir um roteiro, ou linear (Lin), que consiste em uma investigação mais dirigida, com roteiro a seguir;

b) ordem de composição (OC), define a organização das etapas usadas na composição dos conteúdos de um OA, ou seja, a ordem em que essas etapas são apresentadas para o aluno, sendo três ordens de composição definidas, e o que muda de uma ordem para outra é a posição da etapa Resumo, que pode estar no início na composição dos conteúdos, antes da etapa Atividade, ou antes da etapa Avaliação: ordem $1-1^{\circ}$ Introdução, $2^{\circ}$ Desenvolvimento, $3^{\circ}$ Resumo, $4^{\circ}$ Atividade, $5^{\circ}$ Avaliação; ordem $2-1^{\circ}$ Introdução, $2^{\circ}$ Desenvolvimento, $3^{\circ}$ Atividade, $4^{\circ}$ Resumo, $5^{\circ}$ Avaliação; e ordem $3-1^{\circ}$ Resumo, $2^{\circ}$ Introdução, $3^{\circ}$ Desenvolvimento, $4^{\circ}$ Atividade, $5^{\circ}$ Avaliação;

c) ordem de detalhamento (OD), estabelece como os conteúdos no OA podem ser apresentados em nível de particularidades, definida como Específico-para-Geral (Espp-Ger), no qual se inicia na parte específica e prossegue-se para a parte geral para compreensão de um todo; ou Geral-para-Específico (Ger-p-Esp), no qual se inicia na parte geral e prossegue-se para a parte específica para compreensão de um todo;

d) recurso (R), define quais tipos de recursos podem ser utilizados nos elementos da composição de conteúdos para apresentação do OA, os recursos atribuídos na adaptação podem ser Video (Vid), Diagrama (Dia), Gráfico (Gra), Figura (Fig), Narração (Nar), Palestra (Pal), Slide (Sli), Autoavaliação (Aav), Tabela (Tab), Experimento (Exp), Exercício (Exe), Simulação (Sim), Questionário (Que), Esquema (Esq), Animação (Ani), Foto (Fot), Página-Web (Pag), Mapa (Map), Demonstração (Dem), Exemplo (Exem); assim, um conteúdo pode ser composto por mais de um recurso, esses recursos são classificados em relação ao formato de sua apresentação em imagem e (ou) texto.

Os atributos e parâmetros indicam como a interface do OA pode mudar para alunos com diferentes EA. As mudanças ocorrem em relação à (ao):

- sequência ou ordem em que os elementos de composição do conteúdo do OA foram organizados na parte da interface referente ao Índice de Conteúdos, respeitando as três OCs definidas, que alteram a ordem em que esses elementos serão colocados na organização do conteúdo do OA.

- tipos de recursos que podem ser usados na criação do OA. Esses recursos são classificados como texto ou imagem, e foram definidos 20 tipos de recursos que podem ser usados na criação do OA. Os recursos foram organizados na Área de Conteúdos da interface junto com a parte textual do OA. Na parte da interface referente à Área de Conteúdos, os recursos são organizados. 
VII Congresso Brasileiro de Informática na Educação (CBIE 2018)

Anais do XXIX Simpósio Brasileiro de Informática na Educação (SBIE 2018)

- forma de explorar o conteúdo do $\mathrm{OA}$, à maneira como o aluno pode navegar no conteúdo do OA, usando a Área de Controle de Navegação e(ou) o Índice de Conteúdos: se livremente, de forma aleatória, sem seguir um roteiro, ou de forma controlada, com roteiro a seguir, indicado pelo atributo FE.

- nível de detalhamento da apresentação dos conteúdos apresentados, à maneira como estes foram abordados, iniciando do mais específico e prosseguindo para a parte mais geral para compreensão de um todo, ou iniciando na parte mais geral e prosseguindo para a parte mais específica para compreensão de um todo.

A partir dessa estrutura os conteúdos dos OAs podem ser compostos adotando a apresentação adaptada ao EA do aluno, todo o detalhamento do modelo de adaptação criado pode ser consultado em [Silva et al. 2016].

\section{Resultados e Discussão}

A avaliação da adaptação definida foi realizada com base na visão do aluno quanto à qualidade afetiva da interface do OA avaliado durante sua interação com o objeto. Os resultados obtidos das medidas subjetivas das respostas emocionais do aluno em relação à interface do OA indicaram que a adaptação definida atingiu os resultados almejados quanto à adequação da interface do objeto, de acordo com os estilos do Felder and Silverman Learning Style Model - FSLM [Felder and Silverman, 1988].

Para avaliar aspectos relacionados à resposta emocional/qualidade afetiva dos alunos quanto à adaptação da apresentação da interface do OA, o método empírico utilizado foi a aplicação do questionário Self-Assessment Manikin (SAM) [Lang 1985], uma vez que, de acordo com [Zhang 2005], sistemas que despertam sentimentos positivos de afeto são mais regularmente utilizados, mais fáceis de aprender e produzem resultados mais harmoniosos.

\subsection{Descrição do Processo de Avaliação}

Inicialmente, na primeira fase, foi realizado um teste com os alunos selecionados para participarem do processo de avaliação, por meio de formulários online, para identificar o EA. O teste realizado utilizou o Index of Learning Styles - ILS [Felder and Soloman 2006)] do FSLSM como instrumento de mensuração dos EA. Os resultados dos testes (cálculo do EA) foram processados via sistema, para gerar um relatório da identificação do EA, em relação as quatro dimensões de FSLSM.

Com base nesse relatório, foram enviados, na segunda fase, os convites aos alunos para participarem da avaliação do OA adaptado ao seu EA. Foi elaborado para os alunos participantes um formulário online com as instruções da avaliação e a explicação do acesso ao OA que seria avaliado.

\subsection{Participantes}

A amostra desse experimento envolveu, inicialmente, 184 alunos participantes ( $1^{\text {a }}$ fase $)$ dos cursos de bacharelado em Sistemas de Informação, Engenharia Elétrica e Engenharia da Computação, da Universidade Federal Sul e Sudeste do Pará (UNIFESSPA). Todos os alunos dos três cursos foram convidados a participar da pesquisa de maneira voluntária no ano de 2017. 
VII Congresso Brasileiro de Informática na Educação (CBIE 2018)

Anais do XXIX Simpósio Brasileiro de Informática na Educação (SBIE 2018)

Os alunos participantes foram convidados a preencherem um formulário online para identificação dos EA, de acordo com o ILS do FSLSM. Como resultado da primeira fase do processo de validação, foram obtidos 127 formulários preenchidos. $\mathrm{Na}$ etapa seguinte ( $2^{\mathrm{a}}$ fase), foram enviados os formulários online para os 127 alunos participarem da avaliação do OA adaptado ao seu EA. Dos 127 alunos, apenas um não respondeu ao formulário.

\subsection{Resultado da Aplicação do Instrumento ILS}

Os resultados obtidos com a aplicação do questionário online (ILS) para identificação dos EA dos alunos participantes da pesquisa em relação aos estilos identificados do FSLSM são apresentados na Tabela 2. Podemos observar que não há predominância considerável entre os estilos das dimensões. No entanto, se olharmos os valores absolutos, a maioria dos alunos pesquisados apresentou o estilo visual (20 alunos $16 \%$ ), seguida dos alunos com estilo sequencial (18 alunos - 14\%), da amostra de 126 alunos participantes. A dimensão processamento foi a que menos apresentou alunos (14 alunos $-11 \%$ ) para cada um de seus estilos.

Tabela 1. Resultado Questionário ILS para identificação dos estilos dos alunos.

\begin{tabular}{|c|l|c|c|}
\hline \multirow{2}{*}{ Dimensão } & \multicolumn{1}{|c|}{ Estilo } & Quantidade de alunos & Percentual \\
\hline \multirow{2}{*}{ Processamento } & Ativo & 14 & $11 \%$ \\
\cline { 2 - 4 } & Reflexivo & 14 & $11 \%$ \\
\hline \multirow{2}{*}{ Percepção } & Sensorial & 15 & $12 \%$ \\
\cline { 2 - 4 } & Intuitivo & 15 & $12 \%$ \\
\hline \multirow{2}{*}{ Organizaçãõo } & Visual & 20 & $16 \%$ \\
\cline { 2 - 4 } & Verbal & 15 & $12 \%$ \\
\cline { 2 - 4 } & Sequencial & 18 & $14 \%$ \\
\hline \multicolumn{2}{|c|}{ Total } & 15 & $12 \%$ \\
\hline \multirow{2}{*}{ Tobal } & 126 & $100 \%$ \\
\hline
\end{tabular}

No planejamento do processo de avaliação da adaptação, foi estabelecido um número médio de 10 alunos por estilo para prosseguir para a segunda fase. Esse número foi superior para todos os oito estilos do modelo de Felder-Silverman.

\subsection{Resultado da Aplicação do Instrumento SAM}

O questionário SAM foi aplicado aos alunos após eles visualizarem a interface do OA e interagirem com ela. Foram utilizadas as três dimensões do SAM, a "satisfação", "motivação" e "sentimento de controle" de uma pessoa ao utilizar um sistema computacional, cada dimensão é representada por uma escala com valores de 1 a 9 , e o usuário deve escolher aqueles que melhor representam suas emoções.

Os resultados foram obtidos somando-se os valores da avaliação em cada dimensão do SAM. Com isso, é possível fazer uma comparação entre valores positivos - círculos de 6 a 9 na escala do SAM -, neutros - círculo 5 na escala do SAM - e negativos - círculos de 1 a 4 na escala do SAM - de cada dimensão em relação ao OA 
VII Congresso Brasileiro de Informática na Educação (CBIE 2018)

Anais do XXIX Simpósio Brasileiro de Informática na Educação (SBIE 2018)

avaliado. Esses resultados fornecem uma boa indicação da reação do aluno ao objeto em análise, evidenciando sua qualidade afetiva imediata.

Após a interação do aluno com o OA adaptado ao seu EA, foi solicitado que ele preenchesse o questionário SAM e, em seguida, avaliasse o OA com a interface adaptada ao estilo "oposto" ao dele. Feito isso, foi aplicado o questionário novamente após essa interação. Assim, por exemplo, o aluno ativo avaliou o OA adaptado ao estilo ativo e, em seguida, o OA adaptado ao estilo reflexivo, que é o estilo "oposto" ao ativo. Esse procedimento foi realizado para todos os estilos das dimensões do modelo de Felder-Silverman. Desse modo, foi feita uma análise separada dos resultados obtidos nas avaliações com o questionário SAM para os dois OAs analisados pelo aluno. Depois, foi feita a análise comparando os resultados das duas avaliações.

Considerando as avaliações positivas (AV+) e Negativas (AV-), os resultados obtidos por meio da aplicação do questionário SAM para o aluno em relação ao OA adaptado ao seu estilo (por exemplo, aluno ativo avaliando OA ativo), apresentaram mais $\mathrm{AV}+$ que $\mathrm{AV}$ - nas dimensões satisfação (Sa), motivação (Mo) e sentimento de controle (Sc) do SAM, para todos os estilos do FSLSM, conforme demonstrado na Tabela 2. Esses resultados demonstraram que os alunos, em sua maioria, se mostraram satisfeitos, motivados e no controle do OA adaptado ao seu estilo. Com base nesses resultados, pode-se inferir, em princípio, que os resultados em relação aos aspectos de qualidade afetiva demonstraram que o OA estava adequado ao estilo do aluno.

Ainda analisando os dados da Tabela 2, no geral, as avaliações realizadas pelos alunos em relação ao OA "oposto" ao seu estilo (por exemplo, aluno ativo avaliando $\mathrm{OA}$ reflexivo) tiveram mais $\mathrm{AV}$ - que $\mathrm{AV}+$, para as três dimensões do $\mathrm{SAM}$, para os alunos ativos, reflexivos, sequenciais, globais, verbais e visuais. Esses resultados apresentaram indicativos, para afirmar que a reação desses alunos ao OA adaptado ao seu estilo "oposto" foram mais negativas, o que demonstra que não se sentiram motivados, satisfeitos ou no controle em relação a esses OAs. Apenas para os alunos sensoriais e intuitivos as AV- não superaram as AV+. Portanto, esse resultado pode dar indicativos de que a adaptação realizada no OA adaptado ao estilo "oposto" ao desses dois grupos de alunos também os agradou. Esse resultado também pode indicar que a adaptação criada para esses OAs não apresentou mudanças suficientes, em termos de alterações entre o OA adaptado ao estilo correto do aluno e o OA adaptado ao estilo "oposto" ao dele, que pudessem trazer mais reações negativas para esses alunos, caso as alterações não fossem agradáveis a eles.

Conforme mencionado anteriormente, esperava-se que o aluno avaliasse mais positivamente o OA adaptado ao seu estilo e mais negativamente o OA adaptado ao seu estilo "oposto". Caso isso fosse confirmado, seria possível inferir que a adaptação realizada nos estilos de cada dimensão do FSLSM conseguiu refletir mais precisamente as características de cada estilo por meio da adaptação criada para a interface.

Assim, foi definido analisar estatisticamente os resultados das AV- e AV+ realizadas pelo aluno de determinado estilo em relação ao OA adaptado ao estilo "oposto". Essa análise foi feita para todos OAs adaptados para os oito estilos das quatro dimensões do FSLSM. Para realizar a análise estatística, inicialmente definimos duas hipóteses: nula (H0) - os resultados das AV+ e AV- do OA adaptado ao estilo do aluno e do OA adaptado ao estilo "oposto" ao dele não apresentaram diferenças significativas; 
VII Congresso Brasileiro de Informática na Educação (CBIE 2018)

Anais do XXIX Simpósio Brasileiro de Informática na Educação (SBIE 2018)

alternativa (H1) - os resultados das AV+ e AV- do OA adaptado ao estilo do aluno e do OA adaptado ao estilo "oposto" ao dele apresentaram diferenças significativas.

Para avaliar se a diferença de frequências das AV+e AV- observadas é significativa, utilizamos o teste exato de Fisher, com grau de confiança de $95 \%(\alpha=$ $0,05)$. O nível de significância adotado em todo o processo estatístico efetuado foi de $5 \%$. Consideramos estatisticamente significativos valores de $p<=0,05$.

Os resultados do p-value são em relação à frequência de $\mathrm{AV}+\mathrm{e} \mathrm{AV}$ - do $\mathrm{OA}$ adaptado ao estilo do aluno e do OA adaptado ao estilo "oposto" ao dele.

Tabela 2. Resultado geral do SAM para os oito estilos do FSLSM

\begin{tabular}{|c|c|c|c|c|c|c|c|}
\hline \multirow{2}{*}{ Aluno } & \multirow{2}{*}{ OA } & \multicolumn{2}{|c|}{ Satisfação } & \multicolumn{2}{|c|}{ Motivação } & \multicolumn{2}{|c|}{ Sentimento de controle } \\
\hline & & $\mathrm{AV}+$ & $\mathrm{AV}-$ & $\mathrm{AV}+$ & $\mathrm{AV}-$ & $\mathrm{AV}+$ & $\mathrm{AV}-$ \\
\hline \multirow{3}{*}{ ativo } & ativo & 12 & 1 & 11 & 2 & 11 & 1 \\
\hline & reflexivo & 3 & 8 & 3 & 10 & 4 & 8 \\
\hline & p-valor & \multicolumn{2}{|c|}{0,001683} & \multicolumn{2}{|c|}{0,002416} & \multicolumn{2}{|c|}{0,004711} \\
\hline \multirow{3}{*}{ reflexivo } & ativo & 4 & 9 & 3 & 10 & 4 & 10 \\
\hline & reflexivo & 11 & 2 & 11 & 2 & 12 & 2 \\
\hline & p-valor & \multicolumn{2}{|c|}{0,007710} & \multicolumn{2}{|c|}{0,002416} & \multicolumn{2}{|c|}{0,003165} \\
\hline \multirow{3}{*}{ sequencial } & sequencial & 13 & 3 & 13 & 2 & 13 & 2 \\
\hline & global & 4 & 13 & 4 & 12 & 4 & 11 \\
\hline & p-valor & \multicolumn{2}{|c|}{0,001214} & \multicolumn{2}{|c|}{0,000753} & \multicolumn{2}{|c|}{0,001255} \\
\hline \multirow{3}{*}{ global } & sequencial & 4 & 7 & 4 & 8 & 4 & 9 \\
\hline & global & 12 & 1 & 12 & 1 & 12 & 1 \\
\hline & p-valor & \multicolumn{2}{|c|}{0,006057} & \multicolumn{2}{|c|}{0,003258} & \multicolumn{2}{|c|}{0,001804} \\
\hline \multirow{3}{*}{ visual } & visual & 16 & 2 & 17 & 1 & 15 & 1 \\
\hline & verbal & 2 & 15 & 3 & 15 & 4 & 14 \\
\hline & p-valor & \multicolumn{2}{|c|}{0,000005} & \multicolumn{2}{|c|}{0,000002} & \multicolumn{2}{|c|}{0,000027} \\
\hline \multirow{3}{*}{ verbal } & visual & 3 & 9 & 3 & 10 & 2 & 7 \\
\hline & verbal & 11 & 3 & 11 & 2 & 11 & 1 \\
\hline & $p$-valor & \multicolumn{2}{|c|}{0,008931} & \multicolumn{2}{|c|}{0,002416} & \multicolumn{2}{|c|}{0,002167} \\
\hline \multirow{3}{*}{ sensorial } & sensorial & 12 & 1 & 10 & 2 & 10 & 4 \\
\hline & intuitivo & 5 & 7 & 5 & 6 & 6 & 7 \\
\hline & $p$-valor & \multicolumn{2}{|c|}{0,009977} & \multicolumn{2}{|c|}{0,070602} & & \\
\hline & sensorial & 6 & 6 & 5 & 6 & 6 & 7 \\
\hline intuitivo & intuitivo & 12 & 2 & 12 & 1 & 12 & 2 \\
\hline & $p$-valor & 0,0 & & & & & \\
\hline
\end{tabular}

Analisando os resultados do valor de $p$, observamos que, para os alunos ativos, o valor de $p$ foi menor que $0,05(p=0,001683 ; p=0,002416 ; p=0,004711)$ nas três 
VII Congresso Brasileiro de Informática na Educação (CBIE 2018)

Anais do XXIX Simpósio Brasileiro de Informática na Educação (SBIE 2018)

dimensões do SAM. Esse resultado para o valor de $p$ menor que $0,05(p<0,05)$ nas três dimensões do SAM ocorreu também para os alunos com os estilos reflexivo ( $p=$ $0,007710 ; p=0,002416 ; p=0,003165)$, sequencial $(p=0,001214 ; p=0,000753 ; p=$ $0,001255)$, global $(p=0,006057 ; \mathrm{p}=0,003258 ; p=0,001804)$, visual $(p=0,000005 ; p$ $=0,000002 ; p=0,000027)$ e verbal $(p=0,008931 ; p=0,002416 ; p=0,002167)$.

Para os alunos sensoriais, o valor de $p$ foi menor que 0,05 apenas na dimensão Sa $(p=0,009977)$. Para as dimensões Mo $(p=0,070602)$ e Sc $(p=0,172983)$, o valor de $p$ obtido foi maior que $0,05(p>0,05)$. Para os alunos intuitivos, o valor de $p$ foi maior que $0,05(p>0,05)$ apenas na dimensão Sa $(p=0,061236)$. Para as dimensões Mo $(p=0,018307)$ e $\mathrm{Sc}(p=0,037315)$, o valor de $p$ obtido foi menor que $0,05(p<0,05)$.

Diante da análise descritiva dos resultados obtidos com o teste de Fisher, podemos concluir que a hipótese $\mathrm{H} 0$ foi rejeitada para os alunos ativos, reflexivos, sequenciais, globais, visuais e verbais, nas três dimensões do SAM, rejeitada para os alunos sensoriais na dimensão Sa e rejeitada para os alunos intuitivos nas dimensões Mo e Sc, pois em todos esses casos o valor de $p$ foi menor que 0,05 . Portanto, para esses casos, aceita-se H1, o que indica que a adaptação criada para os OAs baseada nos EAs conseguiu imprimir alterações significativas, evidenciadas por meio das reações mais positivas para o OA adaptado ao estilo do aluno e reações mais negativas em relação ao OA adaptado ao estilo "oposto" ao dele.

No entanto, os resultados do teste de Fisher nos quais $\mathrm{H} 0$ foi aceita (e, logo, H1 rejeitada) foram para os alunos sensoriais, nas dimensões Mo $(p=0,070602)$ e Sc $(p=$ $0,172983)$, e também para os alunos intuitivos apenas na dimensão $\mathrm{Sa}(p=0,061236)$, visto que os valores de $p$ obtidos foram maiores que $0,05(p>0,05)$.

Nesse caso, ao aceitar H0, temos indicações de que as adaptações criadas para os OA baseadas nos estilos sensorial e intuitivo não conseguiram imprimir alterações significativas que pudessem ser percebidas pelos alunos sensoriais e intuitivos, portanto a adaptação criada para a interface para esses dois estilos precisa ser mais investigada.

\section{Considerações Finais}

A criação de novas formas de apresentação de conteúdo do OA levando em consideração o EA do aluno pode gerar uma motivação maior no uso desse tipo de recurso educacional, pois o aluno receberia o OA adaptado de acordo com seu estilo. As características mais relevantes do EA foram mapeadas e associadas, com as formas mais adequadas de apresentar o conteúdo do OA para cada EA, criando uma adaptação de apresentação de conteúdo do OA considerando o EA do aluno.

Os resultados obtidos por meio da aplicação do questionário SAM para os alunos, em relação ao OA adaptado aos seus estilos, forneceram uma boa indicação das reações dos alunos ao interagirem com os OAs, o que evidencia que adaptação de apresentação de conteúdo do OA considerando o EA definida em [Silva et al. 2016], foi adequada para todos os estilos do FSLSM, quando avaliados por alunos identificados com esses estilos.

Portanto, espera-se que o modelo possa ser ampliado e utilizado no âmbito educacional para propiciar OAs mais adequados às preferências individuais de 
VII Congresso Brasileiro de Informática na Educação (CBIE 2018)

Anais do XXIX Simpósio Brasileiro de Informática na Educação (SBIE 2018)

aprendizagem do aluno, possibilitando o aumento da motivação e satisfação no uso de OAs adaptados como recurso educacional para a aprendizagem.

\section{Referências}

Aguiar, J. J. B.; Fechine, J. M.; Costa, E. (2014). Estilos Cognitivos e Estilos de Aprendizagem em Informática na Educação: um mapeamento sistemático focado no SBIE, WIE e RBIE. XXV Simpósio Brasileiro de Informática na Educação (SBIE).

Coffield, F., Moseley, D., Hall, E., Ecclestone, K., et al. (2004). Learning Styles and Pedagogy in post-16 Learning: A systematic and Critical Review.

Dorça, F. A., Lima, L. V., Fernandes, M. A., and Lopes, C. R. (2013). A New Approach to Discover Students Learning Styles in Adaptive Educational Systems. Revista Brasileira de Informática na Educação, 21(1):25-2013.

Felder, R. M. and Silverman, L. K. (1988). Learning and Teaching Styles in Engineering Education. Journal of Engineering Education, 78(7):674-681.

Felder, R. M. and Soloman, B. A. (2005). Index of Learning Styles Questionnaire.

Graf, S. (2007). Adaptivity in Learning Management Systems Focussing on Learning Styles. PhD thesis, Vienna University of Technology.

Hamada, A. K., Rashad, M. Z. and Darwesh, M. G. (2011). Behavior Analysis in a Learning Environment to Identify the Suitable Learning Style. International Journal of Computer Science \& Information Technology (IJCSIT), 3(2):48-59.

Keefe, J. (1979). Learning style: An overview. in national association of their relationship. British Journal of Educational Psychology, 67:199-212.

Lang, P. J. (1985). The cognitive psychophysiology of emotion: Fear and anxiety. 30(2): $131-170$.

Sanders, D. A. and Bergasa-Suso, J. (2010). Inferring Learning Style from the Way Students Interact with a Computer User Interface and the WWW. IEEE Transactions on Education, 53(4):613-620.

Silva, Z. C.; Ferreira, L. R.; Pimentel, A. R. (2016). Modelo de Apresentação Adaptativa de Objeto de Aprendizagem baseada em Estilos de Aprendizagem. In: Anais do XXVII Simpósio Brasileiro de Informática na Educação (SBIE), Uberlândia, p. 717-726.

Tidwell, J. (2010). Designing interfaces: Patterns for effective interaction design. O’Reilly Media, Inc.

Valaski, J., Malucelli, A. e Reinehr, S. (2011). Revisão dos Modelos de Estilos de Aprendizagem Aplicados à Adaptação e Personalização dos Materiais de Aprendizagem. XXII Simpósio Brasileiro de Informática na Educação (SBIE), p. 844-847.

Wiley, D. A. (2001). Connecting learning objects to instructional design theory: A definition, a metaphor, and a taxonomy. Utah State University.

Zhang, P. and Li, N. (2005). The importance of affective quality. Communications of the ACM, 48(9):105-108. 